\title{
Encephalitis and Prion disease susceptibility in Polar Bears
}

\author{
Pardis Tabaee Damavandi
}

Ursus Maritimus is a species of marine bear that lives in the Arctic region including North America, Russia, Greenland, but above all in Svalbard (Bear Island) from which the archipelago takes its name and where hunting is wholly prohibited (Figure 1). Currently there are around 20000-31000 polar bears worldwide, and of these, it is estimated that $2500-3000^{(1)}$ populate the Barents Sea in the Spitzbergen region.

The snow-furred arctic mammal can be also found in captivity in temperate climates, for conservation reasons, and it is at those lower temperatures that the immune system of $U$. Maritimus shows enhanced susceptibility to attacks from extraneous infectious agents. Infectivity is, essentially, at its nethermost in the arctic, particularly due to the effects of temperature and of snow, the latter, which increases mood, relaxation and thus boosts the immune system in the polar bear, although infections in the water remain abundant, where risk of amoebiasis is much higher in the arctic regions compared to the temperate counterparts. An elevated Vitamin D rich diet consisting of seals and dolphins, which however are carriers of parasitic zoonoses or of morbillivirus, accompanied by salmon and arctic fish, play also a role in the bear's wellbeing, by preventing this mammal from developing avitaminosis consequently by reducing chances of outbreaks. The consumption of seal and dolphin carcasses is partly also due to the rise in global temperature annually.

At these latitudes it has been found that the risks of developing viral anti-N-methyl-DAspartate (NMDA) receptor encephalitis is increased in the Ursus. The pathology is characterised by hemiparesis (an example is the case of the captive four-year old polar bear 
cub Knut) and seizures, however, Ursidae have also been impacted negatively by neurodegenerative disorders, such as Alzheimer's disease ${ }^{(2)}$ and incidence of Transmissible spongiform encephalopathies (TSEs) cannot still be excluded across the population ${ }^{(3)}$ although $^{2}$ data is not available. The reasoning behind a suspicion to their susceptibility to TSEs may be due to the lifestyle of polar bears who scavenge on seals when food is scarce, but prion structure analysis can reveal predictive information on the matter.

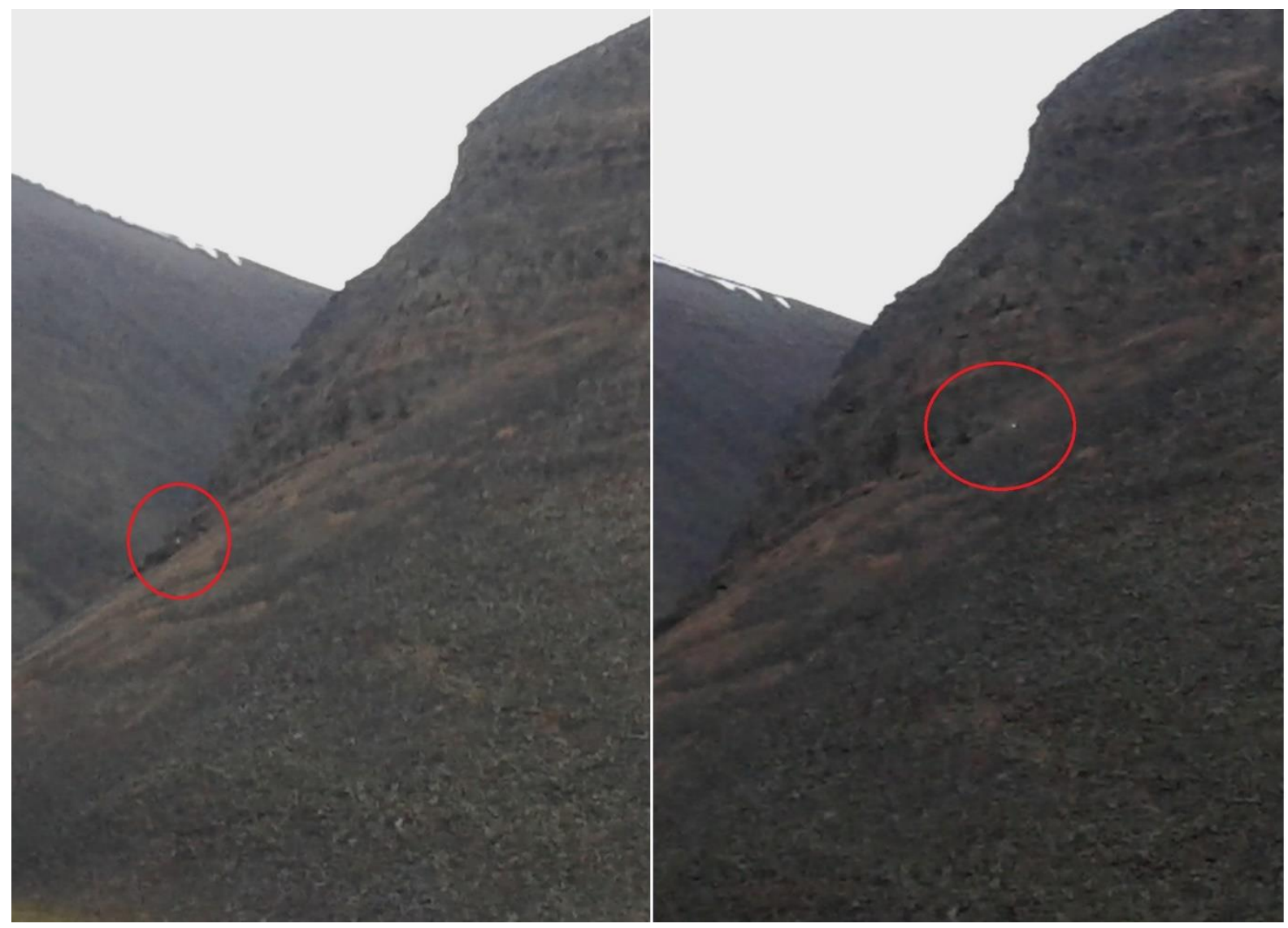

Figure 1. Polar bear cub identified near the Longyearbreen Glacier, moving in the opposite direction to the town of Longyearbyen, Svalbard (Norway); initially mistaken for an arctic fox or arctic hare due to the distance (> $30 \mathrm{~km})$ and inability to spot the mother. The picture on the right signals the polar bear cub leaving the cave at the top of the mountain and potentially chasing the mother opposite the cave and town, but towards the valley where snow is abundant (left photograph); the family may have possibly been disturbed by human vicinity and by the barking of arctic dogs. (Photograph taken in 2015).

\section{ANTI-NMDA RECEPTOR ENCEPHALITIS IN CAPTIVE POLAR BEARS}

A wide range of anaesthetics are normally used to transfer polar bears from one region to another, or into zoos. The most renowned tranquillizer is represented by the drug Reserpine of the alkaloid family Rauwolfia Serpentina which being a natural compound has fewer risks of 
causing the mammal to develop side effects. On a few occasions, however, a Ketamine ${ }^{(4)}$ analogue, Tiletamine, has also been used in combination with a benzodiazepine analogue, Zolazepam. The drug Tiletamine ${ }^{(5)}$ has been known to produce anti-NMDA receptor encephalitis in the human, but Zolapezam could still be used, although withdrawal dangers must be monitored. The use of ketamine analogues should not exclude Encephalitis of differential etiological origins, i.e. fungal or viral. To tell the truth, subarctic regions with high humidity and where stress responses are triggered by unfamiliar climate circumstances for the arctic fauna could be risk factors to emerging fungal infections across the species, even if polar bear genetics makes them quite resistant to viruses.

\section{Investigation of the NMDA receptor (NMDAR) in Thalarctos Maritimus}

A sequence analysis ${ }^{(6)}$ and reconstruction of the glutamate receptor ionotropic NMDA 2A of the polar bear (UniprotKB code: A0A384CJW8) was performed with the use of computational software. The secondary structure (Figure 2) was guessed by comparing it with the homologous ionotropic NMDA receptor structure of the human GluN1/GluN2A (PDB bank code: 6IRH).



Figure 2. Guess of glutamate receptor ionotropic NMDA subunit 2A structure of Ursus Maritimus; Cterminal portion (orange) and the $\mathrm{N}$-terminus (yellow) are highlighted. 
From the sequence analysis (Figure 3) a high homology can be demonstrated among the residues that belong to the Ursid receptor of NMDA, with that of the human, suggesting that not only potential treatment for encephalitis or neurological disorders efficacious on us would also be effective when administered to the bear (allowing opportune dose adjustments), but above all, that pathologies affecting the $\mathrm{Ca}^{++}$-depending receptor could also be compared and studied with the equivalent ones occurring in humans.

Aside of a few variations, the exclusive presence of Cys399 in the polar bear NMDA receptor is of relevance as it operates as a sulphur bridge; equally, the "intrusive" residual blocks, such as "RSNGTVSPSAFLEPFS" (Figure 3), function as "connecting loops" between various motifs providing a more compact and ribbon- or round-like structure to the polar bear 2A subunit. Nevertheless, the NMDA receptor in the human is larger, particularly post-residue 841.

Of lesser importance, throughout the sequence analysis, GV viral-like sequences can be observed, which may support claims that transcriptomics has already identified similarities between the silvery mammal's tissues with retroviruses ${ }^{(7)}$ and that could potentially explain why the polar bear resists to some infectious diseases prevalent in the human.

\section{MGRLGYWTLLVLPALLVWRGPAQGTAAEKGPPALNIAVLLGHSHDVTERELRNLWGPEQAAGLPLDVNVVALLMNRTDP KSLITHVCDLMSGARIHGLVFGDDTDQEAVAQMLDFISSQTFIPILGIHGGASMIMADKDPTSTFFQFGASIQQQATVMLKI MQDYDWHVFSLVTTIFPGYRDFISFIKTTVDNSFVGWDMQNVITLDTSFEDAKTQVQLKKIHSSVILLYCSKDEAVLILSEARS LGLTGYDFFWIVPSLVSGNTELIPKEFPSGLISVSYDDWDYSLEARVRDGLGILTTAAYSMLERFSYIPEAKASCYGQTEKPETP PHTLHQFMVNVTWDGKDLSFTEEGYQVHPRLVVIVLNKDREWEKVGKWENQTLSLRHAVWPRYKSFSDCEPDDNHLSIV TLEEAPFVIVEDIDPLTETCVRNTVPCRKFVKINNSTNEGVNVKKCCKGFCIDILKKLSRTVKFTYDLYLVTNGKHGKKVNNVW NGMIGEVVYQRAVMAVGSLTINEERSEVVDFSVPFVETGISVMVSRSNGTVSPSAFLEPFSASVWVMMFVMLLIVSAIAVF VFEYFSPVGYNRSLARGKAPHGPSFTIGKAIWLLWGLVFNNSVPVQNPKGTTSKIMVSVWAFFAVIFLASYTANLAAFMIQE EFVDQVTGLSDKKFQRPHDYSPPFRFGTVPNGSTERNIRNNYPYMHQYMTKFNQKGVEDALVSLKTGKLDAFIYDAAVLN YKAGRDEGCKLVTIGSGYIFATTGYGIALQKGSPWKRQIDLALLQFVGDGEMEELETLWLTGICHNEKNEVMSSQLDIDNM AGVFYRLAAAMALSLITFIWEHLFY}

Figure 3. Sequence of ionotropic NMDA receptor subunit 2A structure of Ursus Maritimus residues Met1 ( $\mathrm{N}$-terminal region) to Tyr841. In magenta are indicated residues which correspond with those of the Homo Sapiens Sapiens structure, demonstrating that the homology between the inter-species receptors is quite high. Differences are depicted in gray. 


\section{Prion protein of Thalarctos Maritimus}

The sequence of the native prion protein of Thalarctos Maritiums (Figure 4), which was obtained on the Uniprot Database (UniProtKB code: A0A384D3P0), shows again a really high homology with the prion protein of the human (here illustrated the mutant Sporadic Fatal Insomnia type of the human prion protein, PDB code: $1 \mathrm{QLX})^{(6)}$.



Figure 4. Comparison between the native prion protein structure of Thalarctos Maritimus (herewith represented in cyan-blue; the $\mathrm{C}$-terminus is in orange whereas the $\mathrm{N}$-terminus is in yellow) and mutant human prion protein (with the Methionine Met129 mutation) in fuchsia. The blue portions are supplemental to the human prion protein, making the polar bear's prion protein a larger structure. (The Image was obtained with the Pymol visual software) $)^{(6)}$.

Of notice, the longer ancillary arm-like loops extending in opposite directions that seem attached to the isolated native prion protein of Ursus Maritimus (UMR-PrP). Although the structure has been guessed via comparison with the resultant mutant human prion structure (FIPrion), the outcome of heating denaturisation, cooling and minimization calculations performed in parallel, confirms consistent structural stability; this trend was undetected in previous studies on the FI-Prion, characterised on the other hand, by the excessively flexible and disordered residue Methionine (Met129), responsible in producing misfolding and instability across the whole proteic unit. 
Outstandingly, the effect of subjugation of the secondary structure to several techniques leads to the computational endorsement of a helical organisation, as opposed to $\beta$-sheet presentation found in the human prion match. In the latter case, the $\beta$-sheet motif is derived from hydrophobic residues disposed subsequently to each other, i.e. the sequence (Val161-Tyr162Tyr163-Arg164) form one $\beta$-sheet, lost instead as secondary structure in the UMR-PrP, where a lysine residue (Lys164) replaces the Arginine, conferring a standard looping theme, in place of the rigid $\beta$.

Likewise, the initial sequence of Tyr128-Met129-Leu130 sequence providing the second $\beta$ sheet in the FI-prion is entirely absent, and swapped with a long 37 amino acid loop in the UMR-PrP, where the methionine simply represents its N-terminus and is entirely absent within them, whilst the loop is constituted primarily of glycine residues. This arrangement precludes volatility and helps establish structural constancy.

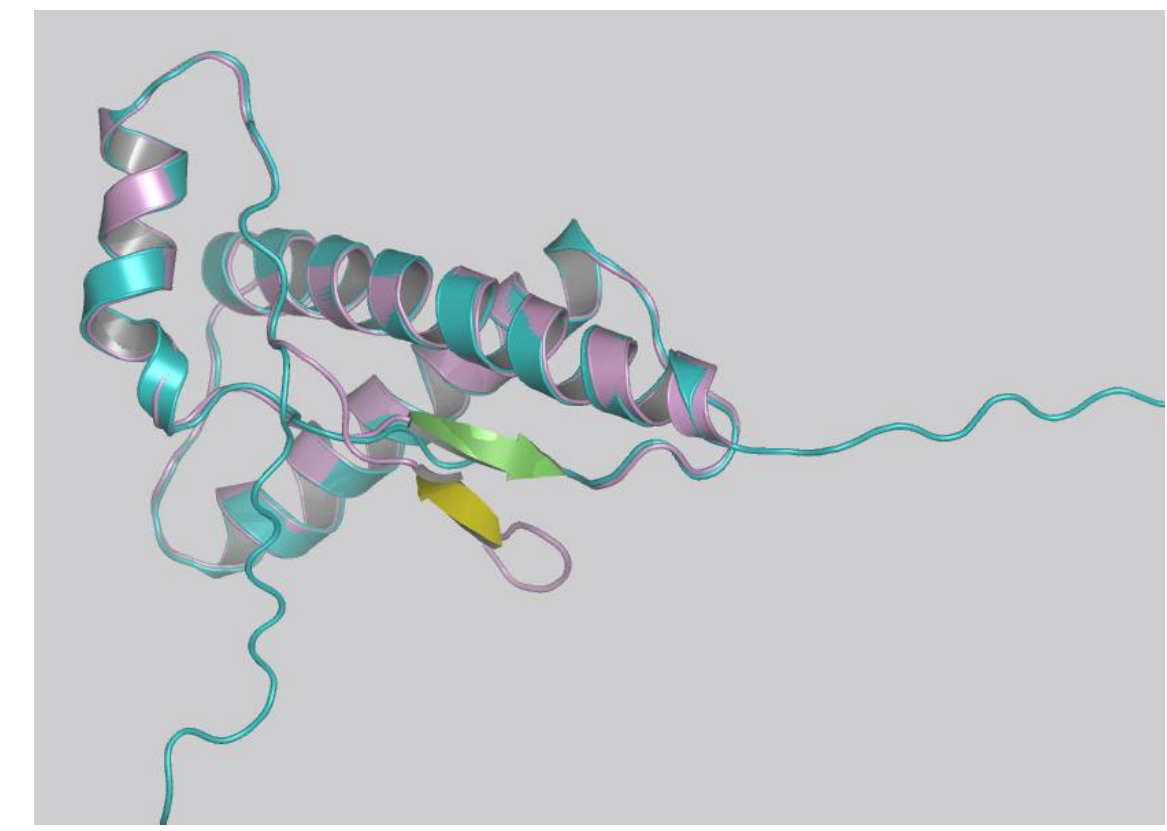

Figure 5. The zoomed structure of the superimposed prion proteins (human in violet, and ursid in teal show the exclusive presence of $\beta$-sheets (detected in Green and Yellow) in the human mutant only. The sequence in the structure. Of Human Fi-prion (violet), the sequence VYYR is illustrated in green, whereas the mutant sequence, MLG is displayed in yellow. The latter occupies an empty space, as the whole sequence is absent in UMR-PrP, which instead possesses two arm-like loops extending from its $\alpha$-helices. 
The Ursid prion also seems more elaborate in size which could explain its additional involvement in ferroptosis (regulated by the PRNP gene) ${ }^{(8)}$. Interestingly, the prion protein in Ursus Maritimus is larger than the sheep prion (scrapie agent) as well as of the human prion protein.

\section{CONCLUSION}

Firstly, this work demonstrates that anti-NMDAR Encephalitis in captive Polar Bears could be caused by the excess use of the Ketamine derivative Tiletamine, because the high homology in the sequences between the NMDA receptor subunit 2A of the Polar Bear and that of the Human outline that both the human and polar bear may show similar pharmacodynamics.

Lastly, the susceptibility of the polar bear to prion disorders may also be conceivable due to the high sequence homology between its native prion protein structure and that once more of the human prion protein, where the only diversity is in the absence of the two $\beta$-sheets, and the elongation provoked by the two extra loop "arms", or branches. The presence of the two loops may make the helices less accessible to extraneous agents, however, the loops would themselves be directly accessible to mutating agents. Moreover, the presence of methionine in other regions (instead of in position 129) with availability within the $\alpha$-helices delineates that susceptibility to mutation of the prion would occur in those positions, therefore Ursus Maritimus susceptibility to Parkinson's Disease or Alzheimer's disease would be more likely, since mutations of prion proteins in these two diseases are $\alpha$-helical in nature.

\section{Acknowledgements}

Special thanks to Polar Bears International (PBI) for map availability of Polar Bear populations and disease incidences across the species. 


\section{Works Cited}

1. Amstrup S. C., DeWeaver, E., Douglas, D. et al. (2010). Greenhouse gas mitigation can reduce sea-ice loss and increase polar bear persistence. Nature, 468, 955-958. https://doi.org/10.1038/nature09653

2. Johnstone E. M., Chaney M. O., Norris F. H. et al., (1991). Conservation of the sequence of the Alzheimer's disease amyloid peptide in dog, polar bear and five other mammals by cross-species polymerase chain reaction analysis. Brain Res Mol Brain Res.; 10(4), 299-305. doi:10.1016/0169-328x(91)90088-f

3. Stewart P., Campbell L., Skogtvedt S. et al., (2012). Genetic predictions of prion disease susceptibility in carnivore species based on variability of the prion gene coding region. PloS one, 7(12), e50623. https://doi.org/10.1371/journal.pone.0050623

4. Santoro J. D., Filippakis A., Chitnis T., (2019). Ketamine use in refractory status epilepticus associated with anti-NMDA receptor antibody encephalitis. Epilepsy Behav. Rep., (12), 100326. doi:10.1016/j.ebr.2019.100326

5. Cording C. J.*, Detuca R., Camporese T. et al, (1999). A Fatality Related to the Veterinary Anesthetic Telazol. J. Analytcal Toxicology, (23), 552-555.

6. Tabaee Damavandi P. (2016), Novel Applications of Lattice Dynamics in the study of protein misfolding and neurodegenerative disorders. Doctoral Thesis, QMUL, London (United Kingdom).

7. Tsangaras K., Mayer J., Alquezar-Planas D. E. et al., (2015). "An Evolutionarily Young Polar Bear (Ursus maritimus) Endogenous Retrovirus Identified from Next Generation Sequence Data." Viruses 7, 11, 6089-6107.

8. Galluzzi L., Vitale I., Aaronson S. A. et al. (2018). Molecular mechanisms of cell death: recommendations of the Nomenclature Committee on Cell Death. Cell Death Differ. 25(3), 486-541. doi:10.1038/s41418-017-0012-4

9. Mano K. K., Matsukawa T., Mitsui J. et al. (2016) Atypical parkinsonism caused by Pro105Leu mutation of prion protein. A broad clinical spectrum. Neurol Genet, 2(1), e48; DOI: 10.1212/NXG.0000000000000048

\section{*Correspondence:}

Dr. Pardis Tabaee Damavandi, E-mail: pardis.tabaee@gmail.com

Copyright: (c) the author(s), publisher and licensee. This is an open-access article distributed under the terms of the Creative Commons Attribution Non-Commercial License, which permits unrestricted non-commercial use, distribution, and reproduction in any medium, provided the original work is properly cited. 